\title{
A highly predictive autoantibody-based biomarker panel for prognosis in early-stage NSCLC with potential therapeutic implications
}

\author{
Akshay J. Patel ${ }^{1}$, Ti-Myen $\operatorname{Tan}^{2}$, Alex G. Richter ${ }^{1}$, Babu Naidu ${ }^{3}$, Jonathan M. Blackburn ${ }^{2,4}$ and Gary W. Middleton (1D ${ }^{1 凶}$ \\ (c) The Author(s) 2021
}

BACKGROUND: Lung cancer is the leading cause of cancer-related death worldwide. Surgical resection remains the definitive curative treatment for early-stage disease offering an overall 5-year survival rate of $62 \%$. Despite careful case selection, a significant proportion of early-stage cancers relapse aggressively within the first year post-operatively. Identification of these patients is key to accurate prognostication and understanding the biology that drives early relapse might open up potential novel adjuvant therapies.

METHODS: We performed an unsupervised interrogation of $>1600$ serum-based autoantibody biomarkers using an iterative machine-learning algorithm.

RESULTS: We identified a 13 biomarker signature that was highly predictive for survivorship in post-operative early-stage lung cancer; this outperforms currently used autoantibody biomarkers in solid cancers. Our results demonstrate significantly poor survivorship in high expressers of this biomarker signature with an overall 5 -year survival rate of $7.6 \%$.

CONCLUSIONS: We anticipate that the data will lead to the development of an off-the-shelf prognostic panel and further that the oncogenic relevance of the proteins recognised in the panel may be a starting point for a new adjuvant therapy.

British Journal of Cancer (2022) 126:238-246; https://doi.org/10.1038/s41416-021-01572-x

\section{INTRODUCTION}

Worldwide, lung cancer is the leading cause of malignancyrelated death in men and the second in women. Only $18 \%$ of patients at initial presentation are suitable for curative treatment, mainly surgical resection. The overall 5 -year survival is 55\%, $35 \%$ and $15 \%$ for stage 1,2 and 3 cancers, respectively $[1,2]$. However, there are still a considerable proportion of patients with resectable lung cancers who relapse very quickly post resection. The behaviour of these cancers does not obey the expected outcomes based on prognostic scores such as the tumour node metastasis (TNM) staging system. The mainstay of treatment for early-stage non-small cell lung cancer (NSCLC) is radical surgery. Stereotactic radiotherapy can be employed for local disease control in patients who are unfit for surgery, but these cases are at higher risk for recurrence [3]. Adjuvant platinum-based chemotherapy has demonstrated an absolute survival benefit of $5 \%$ compared to surveillance alone; however, little progress has been made in this area in the past 10 years. The future of adjuvant therapy will involve multi-modality treatment with targeted molecular agents and immunotherapy [3]. Multimodality therapy is not without associated morbidity; thus, selecting patients who are biologically most at risk of postoperative recurrence is a major clinical need.

Autoantibody (AAb) profiling is a promising approach that incorporates the immune recognition of a myriad of aberrant cancer proteins into a single diagnostic test. AAbs reflect the initial humoral immune response against a tumour and their increased levels can be detectable months to years prior to clinical evidence of a primary tumour [4] or indeed recurrence post resection of a primary tumour. While the mechanisms involved in the production of AAbs in cancer patients remain speculative, AAbs are well known to be sensitive biomarkers in the detection and surveillance of many types of tumours. Gnjatic and colleagues developed protein microarrays to assay the serological response of cancer patients to tumours (serological expression cloning, SEREX) [4]. These high-density protein microarrays, in which proteins are immobilised in their natural conformations, allow the functional testing of thousands of proteins simultaneously, thus increasing the chance of the discovery of new AAb signatures [5]. Building on this work and principle, we utilised the Sengenics Immunome $^{\mathrm{TM}}$ Protein Array [Sengenics, Singapore] containing 1627 proteins, to screen sera from a total of 157 non-small cell

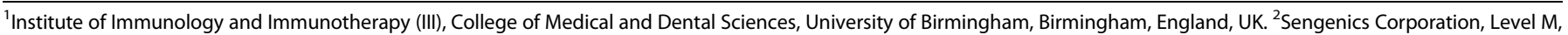

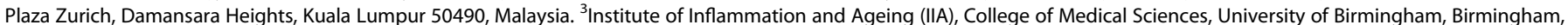

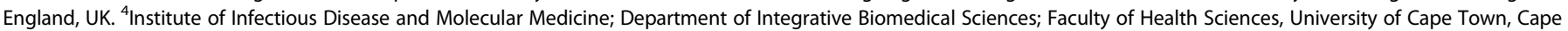
Town, South Africa. ${ }^{凶}$ email: g.middleton@bham.ac.uk 


\begin{tabular}{|c|c|c|c|c|c|c|}
\hline \multirow[b]{2}{*}{ Characteristic } & \multicolumn{3}{|c|}{ Post-operative Mortality } & \multicolumn{3}{|c|}{ Time to Death } \\
\hline & $\mathbf{O R}^{1}$ & $95 \% \mathrm{Cl}^{1}$ & $P$ value & $\mathbf{H R}^{1}$ & $95 \% \mathrm{Cl}^{1}$ & $P$ value \\
\hline Age & 0.97 & $0.94,1.01$ & 0.15 & 0.98 & $0.95,1.00$ & 0.073 \\
\hline IASLC_Stage & 2.19 & $1.43,3.44$ & $<0.001$ & 1.72 & $1.31,2.25$ & $<0.001$ \\
\hline \multicolumn{7}{|l|}{ Vascular_Invasion } \\
\hline No & - & - & & - & - & \\
\hline Yes & 2.28 & $1.15,4.60$ & 0.019 & 2.03 & $1.22,3.37$ & 0.006 \\
\hline
\end{tabular}

Fig. 1 Multivariate analysis using logistic and Cox proportional hazards regression to assess outcome in terms of post-operative mortality and time to death, respectively. Stepwise backward elimination was employed to remove the least significant independent predictors. All variables (age, gender, histology, IASLC stage, nodal status, lymphovascular invasion and adjuvant chemotherapy use) were entered into the model and successively removed depending on significance in the model.

lung cancer (NSCLC) patients across two independent cohorts. We set out to identify a high-risk sub-group of surgically resectable NSCLC patients who may benefit from adjuvant therapy, and explore the biological significance of the identified biomarkers along with information relevant to therapeutic application. We implemented a bespoke machine-learning approach in order to investigate the utility of using the pre-resection samples in the context of malignancy to identify sera-based proteomic changes specifically associated with outcome in NSCLC following surgery.

\section{MATERIALS AND METHODS}

Collaborating clinicians and principal researchers prospectively recruited patients involved in our study across two major tertiary sub-specialty centres in the midlands regions of England, UK as part of a large historical observational study (CLUB) between 2010 and 2015. All patients underwent curative NSCLC (adenocarcinoma or squamous cell carcinoma only) resection (stage I-Illa disease) at two major thoracic surgical units in England. Patients not meeting this inclusion criterion or who had any other previous malignancy were excluded from our study. Inadequate serum sample $(<1 \mathrm{ml})$, non-cancer-related deaths, use of neoadjuvant chemotherapy or positive pathological resection margins were excluded from this study. All participants provided informed consent to participate in future translational studies when they were initially recruited, previously approved by the West Midlands-Solihull Research Ethics Committee (Cancer of the Lung Biomarkers (CLUB): REC reference: 04/Q2704/34). The study had National Cancer Research Network (NCRN) approval and was an NCRN portfolio study. Patients were diagnosed by routine pathological examination of their excised primary tumour and staged according to the TNM staging system for NSCLC according to the International Association for the Study of Lung Cancer (IASLC) guidelines (8th Edition) [6].

\section{Study design}

A total of 157 study participants' (NSCLC stage I-Illa) pre-operative serum samples were utilised in the proteomics analysis, taken from a large repository of trial patients. A sample size calculation, undertaken to achieve a power of $95 \%$ was determined based on the standard deviations of each protein in the immunome array. A random set of patients was selected from the total study participants (investigators were blinded to clinical metadata) in order to train the machine-learning model and subsequently tune the model hyperparameters using $k$-fold cross-validation. This training cohort is known as cohort 1. A smaller independent, second cohort was randomly selected to provide an unbiased evaluation of the final model and validate the model (cohort 2). Cohort sizes were determined using a stratified random sample-based approach to split the overall dataset. For reasonably sized datasets $(n>100)$, this commonly used approach in machine-learning settings has been shown to be close to optimal when allocating $66-70 \%$ of the samples to the training set (cohort 1) [7].

\section{Study cohorts}

Cohort 1 consisted of 111 NSCLC patients 65 survivors, 46 nonsurvivors). Cohort 2 consisted of 46 NSCLC patients (27 survivors, 19 non- survivors). Survivors were defined as patients who were alive and recurrence-free at follow-up. The median follow-up of the entire recurrence-free population was 1825 days (range 1195-2555 days). Non-survivors were defined as patients who died from post-operative recurrence within a median of 365 days. The participant characteristics are summarised in Supplementary Table (S1). There was no significant difference between cohorts 1 and 2 in terms of age, gender, histology, stage, vascular invasion, need for adjuvant therapy and overall survival (assessed using Wilcoxon's rank-sum test and Fisher's exact test for nonparametric continuous data and categorical data, respectively). There was a higher preponderance of adenocarcinomas in cohort 2 (60.9 versus $49.5 \%$ ), and a higher preponderance of squamous cell carcinomas in cohort 1 (50.5 versus $39.1 \%)$. The survival distribution of the total study population is displayed in Fig. 1. Cox proportional multivariate hazards analysis identified the IASLC stage and the presence of lymphovascular invasion as significant independent negative prognostic risk factors (hazard ratio (HR) 1.72, $p<0.001$ and HR 2.03, $p=0.006$, respectively); histology was not significant.

All samples were assayed using the Sengenics Immunome Protein Array containing $1600+$ proteins spotted in quadruplicates (Sengenics, Singapore).

\section{Sample collection}

Serum samples were taken at enrolment or prior to surgery and immediately pseudonymised so as to blind investigators to endpoints. Samples were collected from all participants in a starved state to maintain uniformity. A sample of $7 \mathrm{ml}$ whole venous blood was taken into standard collection tubes and allowed to clot for $2 \mathrm{~h}$. Samples were centrifuged at $3000 \times g$ for $20 \mathrm{~min}$. Serum was then carefully aspirated, divided into aliquots and stored at $-80^{\circ} \mathrm{C}[8]$.

\section{Protein Immunome AAb assay}

Serum samples were thawed, mixed by vortexing and any precipitate was pelleted by centrifugation $(13,000 \times \mathrm{g}$, for $3 \mathrm{~min})$. Aliquots of each sample $(11.25 \mu \mathrm{l})$ were then diluted 400 -fold into Serum Assay Buffer (SAB; 0.1\% v/v Triton, $0.1 \% \mathrm{w} / \mathrm{v}$ bovine serum albumin (BSA) in phosphate-buffered saline; $\left.20^{\circ} \mathrm{C}\right)$, giving a final volume of $4.5 \mathrm{ml}$.

Replica Immunome protein array slides were removed from storage buffer and washed in $200 \mathrm{ml}$ cold SAB on an orbital shaker (50 RPM, 5 $\mathrm{min}$ ). Each slide was then placed array side up in a hybridisation chamber and incubated with individual diluted sera $(4.5 \mathrm{~mL})$ on a horizontal shaker for $2 \mathrm{~h}$ at $20^{\circ} \mathrm{C}$, with gentle agitation. Each protein array slide was then rinsed briefly twice with $30 \mathrm{~mL} \mathrm{SAB}$, followed by immersion in $200 \mathrm{~mL}$ of $S A B$ buffer for $20 \mathrm{~min}$ at room temperature with gentle agitation. Each slide was then incubated with a detection antibody $(20 \mu \mathrm{g} / \mathrm{ml}$ Cy3labelled anti-human $\lg G$ in $S A B$ ) for $2 \mathrm{~h}$ at room temperature with gentle agitation, rinsed briefly with $S A B$ buffer and then washed three times in $\mathrm{SAB}$ for $5 \mathrm{~min}$ at room temperature. Excess buffer was removed by immersing the slide briefly in $200 \mathrm{~mL}$ deionised water, after which slides were then dried by centrifugation $(240 \times \mathrm{g}$ for $2 \mathrm{~min})$ at room temperature. Slides were then stored at room temperature and scanned the same day at $10 \mu \mathrm{m}$ resolution using an Agilent G2505C fluorescence microarray laser scanner.

An outline of the bioinformatics analysis algorithm is shown in Fig. 2. 
Data Pre-processing

Total number of raw candidate biomarkers; $n=1622$

Filtering based on penetrance fold change analysis; $n=1355$

Recursive Feature Elimination with Random Forest Machine

Learning

Repeated cross-fold validation looped over 100 unsupervised iterations

Top most stable predictive biomarkers identified; $\boldsymbol{n}=\mathbf{6 0}$

Corroboration of Marker Selection

Lasso regression

DESEq2

RFE with LOOCV resampling estimation

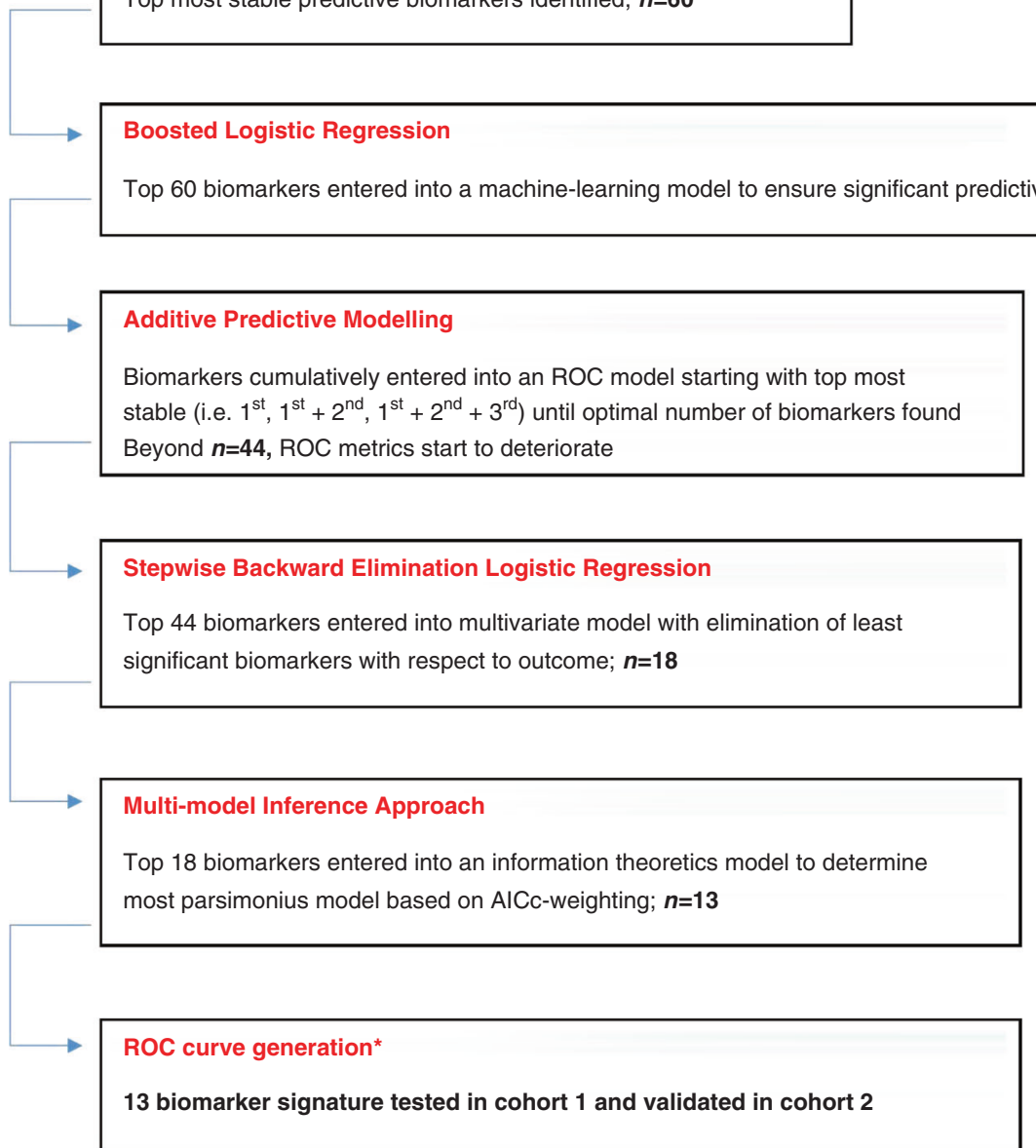

Fig. 2 Data analysis algorithm illustrating steps in raw data handling to applied machine-learning processes and generation and testing of a final biomarker panel in the validation cohort. Throughout the algorithm, we indicate the number of biomarkers that are successively eliminated based on variable importance and stability in the model. At each iterative step, the number of biomarkers is displayed indicating successive removal and refinement of the model.

\section{Data pre-processing}

Scanned images were pre-processed and quality control checks were performed on the generated data using the Sengenics internal pipeline [9]. Composite normalisation of the data was done subsequently by using both quantile- and intensity-based modules on the Cy3-labelled biotinylated BSA-positive control probes as reported by Duarte et al. [10]. AAb binding towards specific proteins was presented as relative fluorescent intensities (RFUs) and used as inputs for downstream analysis.

\section{Penetrance fold-change analysis}

The penetrance fold-change ( $\mathrm{pFC}$ ) analysis compares both the frequency and strength of $A A b$ signals with the intention of identifying biomarkers that are highly elevated in survivors. To achieve this, individual FCs of survivors and non-survivors were estimated using the equation below:

$\mathrm{IFC}_{(\text {protein } \mathrm{A} \text {, sample } \mathrm{X})}=\mathrm{RFU}_{(\text {protein } \mathrm{A}, \text { sample } \mathrm{X})} / \mu \mathrm{RFU} \mathrm{(protein} \mathrm{A,} \mathrm{control} \mathrm{group)}$
Protein A represents each protein in the Immunome array and $\mathrm{X}$ represents every sample assayed in the microarray platform. The mean RFU value for each protein in the control group was used as a background threshold.

For both the survivor and non-survivor groups, respectively, pFC values for each group were obtained by calculating the mean IFC of patients who pass the IFC threshold of $\geq 2$. The penetrance frequencies were then calculated by estimating the number of patients (in each group) who has an IFC $\geq 2$ [11]. Biomarkers were further filtered based on the criteria of (i) pFC of survivors $\geq 2$, (ii) \% penetrance frequency of survivors $\geq 10 \%$ and (iii) penetrance frequency of non-survivors $\leq 10 \%$.

\section{Selection of biomarker panel}

A combination of feature selection and machine-learning methodologies were used to determine the optimal number of biomarkers that were able to provide the best stratification between survivors and non-survivors [12]. For feature selection, univariate statistical tests, random forest importance 
and mutual information metrics were used as filter methods to rank biomarkers (the full list of filter functions are listed in Supplementary Methods (S2)). Given the degree of multi-collinearity between the biomarkers, Recursive-feature elimination (RFE) with random forest modelling was applied to the dataset, looping across 100 unsupervised iterations using random seeds for marker reliability. The topmost stable biomarkers were used to generate biomarker panels by additively selecting the top-ranking biomarkers (top $3.75 \%$ of biomarkers, $n=60$ ) in a cumulative fashion, starting with the most stable biomarker from the RFE set (i.e. $1 \mathrm{st}, 1 \mathrm{st}+2 \mathrm{nd}, 1 \mathrm{st}+2 \mathrm{nd}+3 \mathrm{rd}$ etc). Receiver operating characteristic (ROC) metrics were determined for each additive model and the top-performing combination was taken forward as input to machine-learning models. Any further addition of biomarkers did not lead to significant improvements in model performance, but only further increases in computational time. To determine the biomarker panel performance, ROC, sensitivity and specificity were evaluated and the biomarker panel with the best sensitivity and specificity was deemed the optimal panel to stratify between survivors and non-survivors. For this analysis, Boosted Logistic Regression was performed under default settings using accuracy estimation methods, repeated cross-fold validation and leave-one-out cross-validation [13].

\section{Model selection}

To corroborate marker selection from the RFE algorithm, we used lasso regression with repeated tenfold cross-validation in the training set. This was applied using the $\mathrm{R}$ package glmnet. We set the elastic-net penalty, $a$, that bridges the gap between lasso $(a=1$, the default) and ridge regression $(a=0)$, to 0.9 for numerical stability [14]. Furthermore, we processed proteomics data using DESeq2 (v.4.0.2) software to identify differentially expressed proteins between survivors and non-survivors. A cut-off of gene expression FC of $\geq 2$ or $\leq 0.5$ and a false discovery rate $q \leq$ 0.05 was applied to select the most differentially expressed proteins.

\section{Akaike information criterion}

We adopted a model averaging approach using the Akaike information criterion (AIC) weights $[10,15]$ in order to estimate the in-sample prediction error and thereby the relative quality of the statistical models for a given set of data. We used an information-theoretic approach to calculate the AIC for each model permutation within the top-ranking biomarkers using the glmulti and MuMln packages in order to determine the most parsimonious model with the greatest explanatory predictive power. The AIC is a measure of how well a model fits the data relative to the other possible models given the data analysed and favours fewer parameters [16]. The model with the lowest AIC is the best model approximating the outcome of interest. AIC can be expressed as:

AIC $=-2(\log$-likelihood $)+2 K$,

where $K$ is the number of model parameters and log-likelihood is a measure of model fit. In this study, as $n / K \leq 60$ for sample size $n$ and the model with the largest value of $K$, we used the second-order bias correction version of the AIC (AICC):

AICc $=-2(\log$-likelihood $)+2 K+2 K(K+1) n-K-1$,

$\mathrm{AICC}=\mathrm{AIC}+2 K(K+1) n-K-1$,

where $n$ is the sample size, $K$ the number of model parameters and loglikelihood is a measure of model fit $[15,17]$. From an information-theoretic perspective, the Akaike weights for a particular model can be regarded as the probability or "weight of evidence" that the model is the best model (in a Kullback-Leibler sense of minimising the loss of information when approximating full reality by a fitted model) out of all of the models considered/fitted based on the available dataset $[15,16]$.

\section{RESULTS}

\section{Identification of predictive biomarkers (Fig. 2)}

Initial data processing involved filtering according to the pFC analysis in order to avoid biasing subsequent model generation. One thousand three hundred and fifty-five biomarkers remained, which were taken forward into the deeper analysis. The biomarkers, which appeared most frequently with the highest importance values across 100 randomly seeded iterations, are listed in Supplementary Table (S3). Corroborative regression and genomics analysis methods were performed and indicate the biomarkers, which were common to all analytical techniques. Overall, 60 biomarkers (RFE set) were identified as the most stable with no improvement in predictive performance beyond this number.

\section{Additive predictive modelling}

The RFE set of biomarkers was used to generate biomarker panels by additively selecting the top-ranking biomarkers in a cumulative fashion. These inputs were used to determine the ROC metrics at each additive iteration for cohort 1, displayed in Supplementary Graph (S4). An upward linear trend in all three parameters (area under the curve (AUC), sensitivity, specificity) was noted as more biomarkers were added. This progressive increase peaked at 44 cumulative biomarkers (AUC 0.975; sensitivity $87 \%$; specificity 98.5\%). Beyond this, the predictive metrics become rather unstable and less uniform, hence the decision to proceed with the top 44 biomarkers for deeper analysis.

\section{Multi-model inference approach}

Given that a 60-biomarker diagnostic scoring system would be cumbersome and impractical, we utilised an information-theoretic approach to determine the biomarker combination with the highest diagnostic potential in the most parsimonious model. We employed the AICc method in order to estimate the "goodness of fit" of statistical models and thereby compare multiple models with one another. The AICC avoids overfitting the model in smaller sample sizes. Based on the cumulative ROC analysis, we proceeded with the top 44 biomarkers in this downstream analysis. Following stepwise backward elimination of these markers in a multivariate logistic regression model, with survivorship as the dependent variable, 18 biomarkers were determined to be the most significant and were therefore used in the multimodel inference analysis. Any further addition of more biomarkers did not lead to significant improvements in model performance, but did contribute to significant increases in computational time.

\section{Assessing model performance}

Panel $a$, the most parsimonious and best-performing model, comprised 13 biomarkers-SPATA19, TSPY3, GLS2, TCEA2, TSGA10, HMGN5, LUZP4, HDAC4, SPACA3, IMPDH1, TXN2, TFG and PPP2R1A (Supplementary Table (S5)). ROC metrics for each individual candidate biomarker are found in Supplementary Table (S6), along with association with clinico-pathological correlates (Supplementary Figure S7). This refined model was assessed in cohort 1 (AUC 0.918 , sensitivity $89.1 \%$, specificity $80.1 \%$ ) and validated in the independent cohort 2 (AUC 0.842 , sensitivity $84.2 \%$, specificity $74.1 \%$ ) (Fig. 3). There was no significant difference in the ROC metrics between the two cohorts, indicating good performance in the validation cohort. We noted a preponderance of bona fide cancer testis antigens (CTAGs) in the RFE biomarker set $(16 / 60$ (26.7\%)). We thus elected to explore two further CTAG specific panels in order to determine the prognostic relevance of these highly conserved proteins in NSCLC. We refer to the final biomarker panel we defined as panel a (13 biomarkers). Panel $b$ refers to the CTAGs extracted from the RFE set (16 biomarkers) and panel $c$ refers to the CTAGs extracted from panel a (6 biomarkers). The strong CTAG presence in panel a comprises six proteins antigens, SPATA19, SPACA3, TSPY3, TCEA2, TSGA10 and LUZP4, all with established protumourigenic roles in different cancers under certain conditions (Supplementary Table (S5)). CTAGs trigger unprompted humoral immunity and immune responses in malignancies, altering tumour cell physiology and neoplastic behaviours. Their limited expression in normal somatic tissues coupled with recurrent up-regulation in epithelial carcinomas makes them highly attractive biomarker and vaccine targets. We explored the performance of all three panels in 


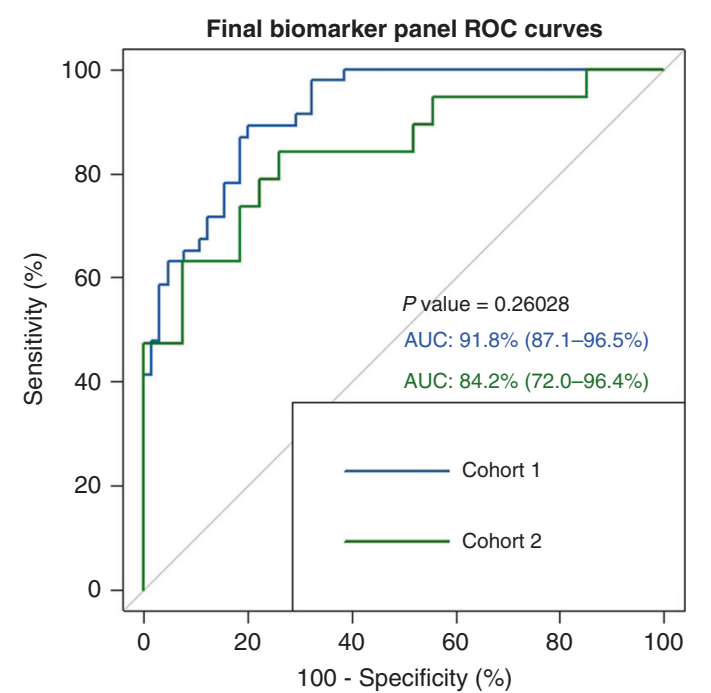

Fig. 3 ROC curves for Biomarker Panel A in both Cohorts. ROC curves demonstrating the performance of panel (a) (13 biomarkers) in both cohort 1 (test) and cohort 2 (validation). $P$ value indicates no significant difference in the performance of this model between cohorts. AUC 95\% confidence intervals are displayed within brackets.

cohorts 1 and 2 (Fig. 4). Panel a performed significantly better in cohort 1 (test) than both panels $b$ and c (CTAG panels). However, in cohort 2 (validation), the differences between panel a and the CTAG panels (b and c) was not significant. Panel b (16 CTAG panels) outperformed panel a in cohort 2 (AUC 0.875 versus $0.842, p=N S$ ), but panel $c$ underperformed compared to panel a in cohort 2 (AUC 0.69 versus $0.842, p=\mathrm{NS}$ ). The increased predictive performance of panel $b$ (16 CTAG panels) reaffirms the importance of CTAGs in discriminating between survivorship in lung cancer. In spite of CTAG preponderance, these data show that the non-CTAG antigens in panel a, which are critical mediators of Wnt signalling and phosphatase activity, are clearly biologically important in their ability to prognosticate in lung cancer.

\section{Survival analysis}

Further interrogation of these signatures was carried out by generating a continuous risk score for every individual on the basis of model coefficients. The resultant predicted risk scores from cohort 1 (training) were divided using optimal cut-off points determined through ROC analysis in order to further dichotomise the patient cohorts as "high expressors" and "low expressors". We performed this for all three panels $(a-c)$, the scores being inferred directly from the biomarker signal intensities. Using these individual risk scores, we carried out survival analyses (Fig. 5) and multivariate Cox proportional hazards modelling (Fig. 6) in the entire NSCLC cohort. Patient age, gender, histology, nodal status, IASLC stage, lymphovascular invasion and whether patients underwent adjuvant chemotherapy were all predictors that were entered into the model alongside all the panel scores. All panels were able to effectively dichotomise between survivor statuses in our cohort, with high expression conferring a significantly worse outcome $(p<0.001)$, reaffirming findings from the ROC analysis. Five-year survival in high expressers of panels $a, b$ and $c$ was $7.6 \%$, $16.4 \%$ and $19.9 \%$, respectively, and high expressers of panel a had a median survival of just under 16 months, which for early-stage resected lung cancer is very low. On multivariate testing, only panels $a$ and $b$ were deemed significant independent predictors of survival, HR 19.6 and 7.22 , respectively $(p<0.05)$. IASLC stage was still deemed an independent predictor of outcome albeit not significant (HR 1.24, $p=0.11$ ). Panel c was deemed a significant independent predictor of outcome only when entered into a multivariate model without panels $a$ and $b$. This reaffirmed the findings that the CTAGs alone from panel a were not sufficiently predictive enough when compared with panels $a$ and $b$, but are still significant predictors independent of age, gender, IASLC stage, lymphovascular invasion, histology, nodal status and whether patients underwent adjuvant chemotherapy.

We performed multivariate analyses in various subgroups according to gender, histology ISALC stage and adjuvant therapy status to explore the relevance of panel a in relation to specific clinico-pathological factors. In all subgroups, panel a was the most significant independent predictor of outcome (Supplementary Table (S8)).

\section{DISCUSSION}

Results from the NLST and European NELSON trials were strongly supportive of lung cancer screening $[18,19]$. Widespread use of CT coronary angiography to assess inpatient chest pain as well as the use of whole-body CT use in the assessment of Trans-catheter valve intervention results in a high detection rate of incidental findings, a large proportion of which are lung malignancies. The combination of screening strategies and increased use of CT scanning for non-cancer-related conditions will result in a surge in the detection rate of early-stage lung cancers and therefore an increased surgical resection rate. Early-stage lung cancers confer a multitude of outcomes ranging from indolent disease with high post-operative disease-free survival rates at 5 years to highly aggressive disease with relapse in the first 12 months post resection.

Current prognostic biomarkers for early-stage lung cancers have been described but are limited in their utility owing to the lack of proper validation and lack of adequate sensitivity and/or specificity. The key points in evaluating biomarker studies in early-stage lung cancer include well-defined objectives and study populations, robust specimen storage and use, and the use of a clinically applicable assay that is validated in an independent cohort of patients. Critical appraisal of published prognostic signatures in early-stage lung cancers found that adherence to these criteria was poor with overt flaws in study design. Subramanian and Simonet al published a set of guidelines to inform prognostic biomarker studies in lung cancer, and although all the studies pertain to gene expression microarray data, we have adhered to these criteria as closely as possible [20]. We validated our signature in one completely independent dataset, which is a feature that is lacking in many prognostic signatures in early-stage lung cancer [20]. A 14-gene, quantitative real-time PCR-derived expression signature was previously validated in two independent large stage I, non-squamous NSCLC datasets, which demonstrated the robustness of the statistical design. This signature showed poor survival in high-risk patients based on gene expression in both validation cohorts, and although the AUC values were significantly higher for this signature than standard NCCN risk criteria in both validation sets, the absolute values were still relatively low (0.60 and 0.61$)$, compared with the AUC values from our panels $a$ and b (AUC 0.842 and 0.875) [21]. Furthermore, this study did not assess the therapeutic relevance of the genes identified in the final signature.

Historically, the majority of AAb-based biomarker research, including that in NSCLC, has concentrated on the diagnosis of disease states or early detection of cancers as opposed to trying to map the course of disease post treatment [22]. Sensitivities and specificities of biomarker panels for lung cancer detection have ranged from 0 to $92.2 \%$ and 79.5 to $92.2 \%$, respectively [23].

Circulating proteins have also been investigated as prognostic biomarkers in early-stage lung cancer, the most common of which are CEA and CYFRA 21-1. The largest study exploring the role of CEA found that elevated pre-operative levels conferred poor 5-year survival [24]. 

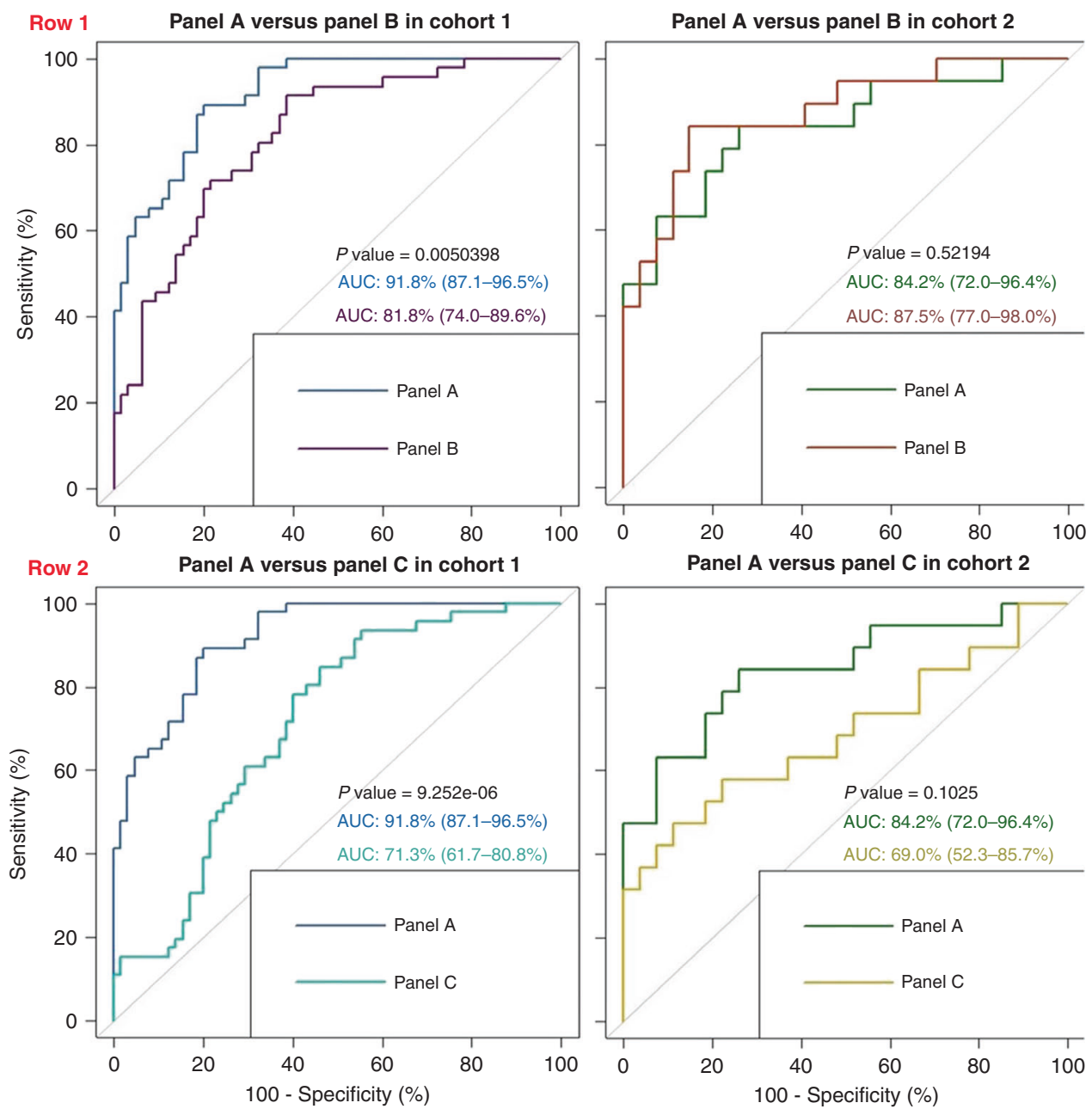

Fig. 4 Comparative ROC curves for Biomarker Panel combinations in both Cohorts. ROC curves demonstrating performance of panel (a) (13 biomarkers) and panel (b) (16 CTAG biomarkers from RFE set) in cohort 1 (test) (row 1, left panel) and cohort 2 (validation) (row 1, right panel. AUC confidence intervals are displayed within brackets. $P$ values indicate significant differences in performance in cohort 1 but not cohort 2. ROC curves demonstrating performance of panel (a) (13 biomarkers) and panel (c) (6 CTAG biomarkers from panel (a)) in cohort 1 (test) (row 2, left panel) and cohort 2 (validation) (row 2, right panel). AUC confidence intervals are displayed within brackets. $P$ values indicate significant differences in performance in cohort 1 but not cohort 2 .

Given the complexity and multi-factorial nature of the antitumour immune response and tumour immune evasion mechanisms in cancers that are not solely reliant on single oncogenic drivers, combination biomarker signatures are more valuable [23]. None of the prognostic studies offered any predictive assessment of their panels, but instead used hazard ratios (measures of association, not predictive power) with no separate test/validation.

Three broad categories of genes comprised our final panel (panel a), namely CTAG expression, Wnt signalling protein aberrancy and serine/threonine protein phosphatase deregulation. CTAGs are united by their role in embryonic development and restriction of expression to male germ cells. Ectopic reexpression of these antigens has been seen in a variety of somatic solid tumours and in triple-negative breast cancers, high expression is correlated with worse survival in multivariate analysis (HR 2.02, 95\% confidence interval 1.27-3.20; $p=0.003$ ) [25]. Ectopic gene signatures of normally silenced CTAG genes that are expressed in cancer associated with a highly aggressive lung cancer phenotype and independently predicted poor outcome [26]. We identified 16 CTAGs (27\%) in our RFE set (S3) as being highly discriminatory for survivorship in this distinct cohort of NSCLC patients (SPATA19, SPACA3, TSGA10, TSPY3, LUZP4, TCEA2, CTNNA2, MAGEB2, SPO11, MAGEB4, MAEL, CSAG1, MAGEB5,
COX6B2, GAGE2 and TSSK6). This CTAG only model displayed high predictive power in the validation cohort (AUC 0.875, sensitivity $84.2 \%$ ) and was a significant independent predictor of poor outcomes. Clonal and subclonal CTAG expansion is generally uniform in tumour cells with variations in behaviour tightly regulated by epigenetic alterations [27].

Aberrant activation of the Wnt/ $\beta$-catenin signalling pathway is causally linked to cancer recurrence, immune evasion and metastasis. A number of the identified tumour-associated antigens are known to signal via this cascade, HMGN5, TFG, MAEL, SOX15 and Dicckopf-1, the latter of which has been investigated in numerous other biomarker signatures [28-30] and like TFG interacts via the Wnt co-receptor LRP6 [31]. Proteins such as MAEL and PTK7 both signal via this cascade and the expression was significantly associated with poor outcomes in our cohort. MAEL, also a CTAG, has been shown to be critical for cancer cell survival and is over-expressed in the bladder and gastric cancers [32]. Functional experiments have determined that MAEL protein exerts its oncogenic dominance through degradation of the protein phosphatase ILKAP [33, 34]. Mutating or silencing of phosphatase activity is a well-known tumour escape mechanism $[35,36]$ and is the third core component of our identified biomarker panel. MAEL provides a 

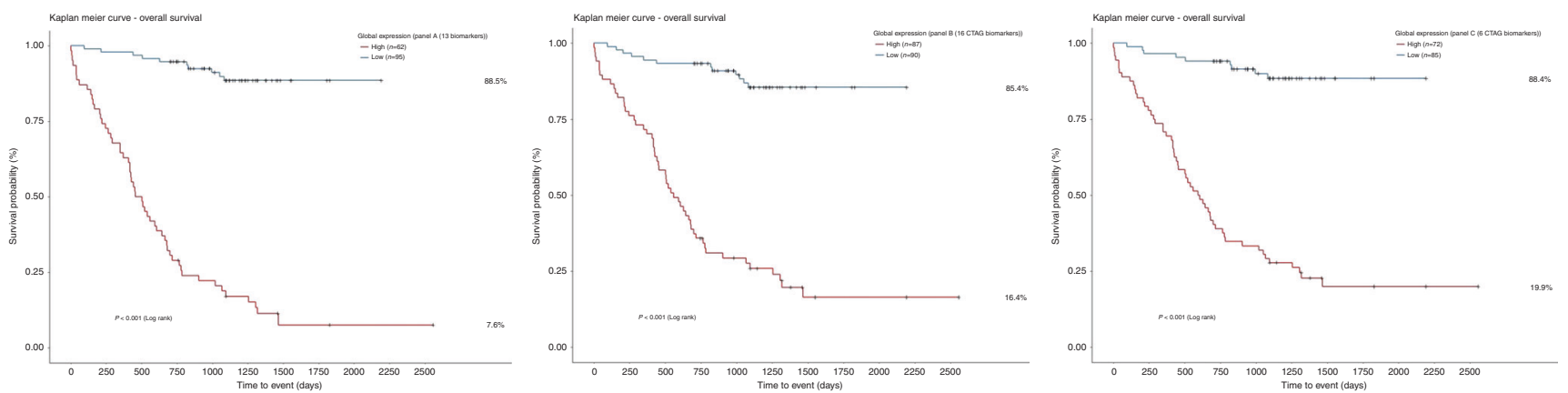

Fig. 5 Kaplan-Meier Survival Curves. Kaplan-Meier survival analysis for panel (a) (13 biomarkers), panel (b) (16 CTAG biomarkers from RFE set) and panel (c) (6 CTAG biomarkers from panel (a)). All curves demonstrate significantly worse outcomes in patients with high global expression of each biomarker signature. The number of patients in each expression group is shown within brackets in the legend for each Kaplan-Meier plot. Percentage survival out to 5 years is displayed to the right-hand side for each expression group in each plot. The median survival for high expressers of panels (a, b and $\mathbf{c}$ ) is 479,558 and 600.5 days, respectively.

unique link between all three biological pathways. These molecules provide unique therapeutic targets as demonstrated by an antibody-drug conjugate against the Wnt signalling PTK7 tyrosine kinase molecule, which elicited potent anti-tumour activity in low-passage patient-derived solid tumour xenograft models [37]. In solid tumours like NSCLC, high PTK7 expression confers significantly reduced overall survival [37]. Targeting this molecule in phase I trials have just completed accrual [NCT02222922]. Multiple other agents targeting the Wnt signalling axis that have entered or completed phase I clinical trials and include Vantictumab, a monoclonal antibody against the Fzd receptor (NCT01345201, NCT02005315, NCT01957007 and NCT01973309), decoy receptors such as OMP-54F28 (NCT02069145, NCT02092363, NCT02050178 and NCT01608867) and porcupine enzyme inhibitors (NCT01351103 and NCT02521844) [28, 38].

Cellular responses to DNA damage are integral to maintaining the genome and preventing cancer progression; serine-threonine phosphatases like protein phosphatase 2 play a key role in the DNA damage response through the regulation of important cell cycle proteins and tumour suppressor genes such as ATM, Chk1, Chk2, p53 and BRCA1 [36]. Cancer cells tend to evade the activation of DNA repair pathways through copy number alterations of Ser/Thr phosphatases, missense mutations and increased mutant gene expression. Identifying aberrancy of these important proteins and utilising early antigen expression is key to disease surveillance and therapeutics. Following the exploitation of $B C R / A B L$ kinase inhibition in chronic myeloid leukaemia, efforts have been made to explore PP2A phosphatase reactivation/ inhibition in anti-tumour therapy. PPP2R1A dysregulation was individually a significant independent predictor of poor survival in both cohorts; belonging to the PP2A enzyme family, these complexes exert control over oncogenic signalling pathways (MEK/ERK and Srk-Jnk) and over collateral resistance phosphorylation pathways. Their inhibition in a KRAS-mutant human lung cancer cell line resulted in improved responses with MEK inhibitors [39]. Mutations of PPP2R1A significantly enhance cancer cell migration in endometrial and ovarian carcinomas [40], whereas allosteric activation of this wild-type complex induces cell cycle arrest with broad anti-tumour activity [35]. Notably, overexpression and mutation of the target antigen are two of the classical mechanisms for AAb production, with CD4 ${ }^{+}$T-helper cells specific to mutated neoepitopes being able to drive the expansion of a set of antigen-specific $B$ cells, resulting in the secretion of polyclonal AAbs that are able to recognise both mutated and wildtype forms of the antigen [41]. Thus, our observation that antiPPP2R1A AAbs are independent predictors of poor survival is consistent with the known aberrant function of PPP2R1A in oncogenesis, albeit our data does not allow us to yet distinguish between the two possible molecular origins of the AAbs. Current phase 2 trials in recurrent glioblastoma (NCT03027388) are investigating the role of PP2A inhibitor, LB100.

Whilst the aim of this study was to identify a highly prognostic panel for surgically resectable lung cancer, the biology of the final markers suggests a line of sight to the clinic in terms of adjuvant therapies, in which high expression of our prognostic biomarker might indicate a very poor outcome group of patients whose survival might be improved by targeting some of the proteins that the AAbs have formed against particularly the CTAGs. The highly restricted expression patterns of CTAGs in normal tissues and ectopic expression in tumour types makes them highly sought after as targets for cancer vaccines [31]. The Lipo-MERIT trial demonstrated strong $\mathrm{CD}^{+}$and $\mathrm{CD}^{+}{ }^{+}$cell induction along with durable objective clinical benefit in unresectable melanoma patients treated with a poly-antigenic liposomal RNA vaccine with or without combination with anti-PD1 checkpoint blockade therapy [32]. The RNA vaccine targeted four main CTAGs: NY-ESO1, MAGEA3, TPTE and Tyrosinase [32]. In our dataset, low CTAG expressers (Fig. 5) had good outcomes, with $85.4 \%$ 5-year overall survival $(p<0.001)$; targeting this group is unlikely, therefore, to be of benefit; however, high expressers who suffer poor outcomes post resection may well be suitable for a CTAG-based polyepitopic RNA vaccine as an adjunct to standard adjuvant chemotherapy in order to further eliminate micro-metastatic deposits and cells with a high biological propensity for aggressive disease.

\section{LIMITATIONS AND FURTHER WORK}

Overall study limitations include the retrospective design and heterogeneity of the study population, which can introduce selection bias. This allied with the clinical diversity of the population may mean the results are less easy interpretable. However, we sought to mitigate against this using our robust random machine-learning-based approach. Despite good performance, the panel identified should also be employed to determine clinical utility in larger independent NSCLC cohorts with the parsimonious panel as well as in other cancers to ascertain if the signature is disease-specific.

There are additional intrinsic limitations related to the identification of proteins markers in biological fluids, in particular the expression of AAb against highly conserved intracellular tumour markers or in immuno-privileged sites. The host immune response against cancer antigens is complex and tends to direct itself towards the most immunogenic epitopes. It is known that autoantigens that are modified before or during the course of tumour formation and progression in cancer can stimulate the immune response in patients when they are released from tumour cells and that immune responses have been observed to be 


\begin{tabular}{lccc}
\hline Characteristic & $\mathbf{H R}^{1}$ & $\mathbf{9 5 \%} \mathbf{C l}^{1}$ & $\boldsymbol{P}$ value \\
\hline 'Panel A' & 19.6 & $4.73-81.6$ & $<0.001$ \\
\hline 'Panel B' & 7.22 & $1.23-42.4$ & $\mathbf{0 . 0 2 5}$ \\
\hline IASLC_Stage & 1.24 & $0.95-1.62$ & 0.11 \\
\hline
\end{tabular}

${ }^{1} \mathrm{HR}$ hazard ratio, $\mathrm{Cl}$ confidence interval.

Fig. 6 Multivariate Cox proportional hazards modelling in entire NSCLC cohort $(n=157)$. Stepwise backward elimination was employed to remove the least significant independent predictors.

responsible for tumour growth promotion, but also prevention in a process called immuno-editing [42]. Further underscoring this, most of the seroreactive biomarkers in the RFE set $(n=60)$ are intracellular antigens $(52 / 60)$ interacting with membrane and nonmembrane-bound organelles such as ribosomes (4/60), with the majority residing within the nucleus (37/60), a usually immunoprivileged site. This pattern has been observed in AAb studies in melanoma [42]. Despite this, AAbs generated against autologous nuclear antigens are frequently found in cancer patient sera [43]. Nuclear antigens, however, do not undergo antigen presentation during the negative selection of self-reactive lymphocytes largely because of their intrinsic proteolytic instability, which affects the binding kinetics with major histocompatibility complex class II receptors. Exposure of the nuclear antigens to one's immune system and the resultant generation of AAbs is, therefore, thought to occur following tumour cell death and release of the intracellular contents into the circulation, although altered cellular localisation [44], or shedding in exosomes, in transformed cells may also play a role. The understanding of these key points might help to clarify the response of our body against cancer autoantigens in a patient-specific manner, but further clinical validation is needed in order to extend the use of these 13 biomarkers in early detection and mapping the prognosis of cancer.

\section{DATA AVAILABILITY}

The datasets generated during and/or analysed during the current study are available from the corresponding author on reasonable request.

\section{CODE AVAILABILITY}

The authors declare that the code for reproducibility of data are publicly available or will be available upon request.

\section{REFERENCES}

1. Ferlay J, Shin H-R, Bray F, Forman D, Mathers C, Parkin DM. Estimates of worldwide burden of cancer in 2008: GLOBOCAN 2008. Int J Cancer. 2010;127:2893-917.

2. International Early Lung Cancer Action Program Investigators, Henschke $\mathrm{Cl}$, Yankelevitz DF, Libby DM, Pasmantier MW, Smith JP, et al. Survival of patients with stage I lung cancer detected on $C T$ screening. $N$ Engl J Med. 2006;355:1763-71.

3. Indini A, Rijavec E, Bareggi C, Grossi F. Novel treatment strategies for early-stage lung cancer: the oncologist's perspective. J Thorac Dis. 2020;12:3390-8.

4. Gnjatic S, Wheeler C, Ebner M, Ritter E, Murray A, Altorki NK, et al. Seromic analysis of antibody responses in non-small cell lung cancer patients and healthy donors using conformational protein arrays. J Immunol Methods. 2009;341:50-8.

5. Ramachandran N, Raphael JV, Hainsworth E, Demirkan G, Fuentes MG, Rolfs A, et al. Next-generation high-density self-assembling functional protein arrays. Nat Methods. 2008;5:535-8.

6. Goldstraw P, Chansky K, Crowley J, Rami-Porta R, Asamura H, Eberhardt WEE, et al. The IASLC Lung Cancer Staging Project: Proposals for Revision of the TNM Stage Groupings in the Forthcoming (Eighth) Edition of the TNM Classification for Lung Cancer. J Thorac Oncol. 2016;11:39-51.

7. Dobbin KK, Simon RM. Optimally splitting cases for training and testing high dimensional classifiers. BMC Med Genomics. 2011;4:31.
8. Rathinam S, Ward DG, James ND, Rajesh PB. Proteomic analysis of resectable nonsmall cell lung cancer: post-resection serum samples may be useful in identifying potential markers. Interact Cardiovasc Thorac Surg. 2011;13:3-6.

9. Sumera A, Anuar ND, Radhakrishnan AK, Ibrahim $\mathrm{H}$, Rutt $\mathrm{NH}$, Ismail NH, et al. A novel method to identify autoantibodies against putative target proteins in serum from beta-thalassemia major: a pilot study. Biomedicines. 2020;8:97.

10. Duarte J, Serufuri J-M, Mulder N, Blackburn J. Protein function microarrays: design, use and bioinformatic analysis in cancer biomarker discovery and quantitation. In: Wang $X$, editor. Bioinformatics of human proteomics. Dordrecht: Springer; 2013. p. 39-74. https://doi.org/10.1007/978-94-007-5811-7 3. Accessed 6 March 2021.

11. Mak A, Kow NY, Ismail NH, Anuar ND, Rutt NH, Cho J, et al. Detection of putative autoantibodies in systemic lupus erythematous using a novel nativeconformation protein microarray platform. Lupus. 2020;29:1948-54.

12. Bommert A, Sun X, Bischl B, Rahnenführer J, Lang M. Benchmark for filter methods for feature selection in high-dimensional classification data. Comput Stat Data Anal. 2020;143:106839.

13. Wright MN, Ziegler A. Ranger: a fast implementation of random forests for high dimensional data in C++ and R. J Stat Softw. 2017;77:1-17. https://doi.org/ 10.18637/jss.v077.i01.

14. Tibshirani R. Regression shrinkage and selection via the Lasso. J R Stat Soc Ser B. 1996;58:267-88.

15. Burnham KP, Anderson DR. Multimodel inference: understanding AIC and BIC in model selection. 2004. https://journals.sagepub.com/doi/10.1177/0049124104268644. Accessed 6 March 2021.

16. Akaike $\mathrm{H}$. Information theory and an extension of the maximum likelihood principle. In: Parzen E, Tanabe K, Kitagawa G, editors. Selected papers of Hirotugu Akaike. Springer Series in Statistics. New York: Springer; 1998. p. 199-213.

17. Cooper JD, Han SYS, Tomasik J, Ozcan S, Rustogi N, van Beveren NJM, et al. Multimodel inference for biomarker development: an application to schizophrenia. Transl Psychiatry. 2019;9:1-10.

18. Yousaf-Khan $U$, van der Aalst C, de Jong PA, Heuvelmans M, Scholten E, Lammers $\mathrm{J}-\mathrm{W}$, et al. Final screening round of the NELSON lung cancer screening trial: the effect of a 2.5-year screening interval. Thorax. 2017;72:48-56.

19. Horeweg N, Scholten ET, de Jong PA, van der Aalst CM, Weenink C, Lammers JWJ, et al. Detection of lung cancer through low-dose CT screening (NELSON): a prespecified analysis of screening test performance and interval cancers. Lancet Oncol. 2014;15:1342-50.

20. Subramanian J, Simon R. Gene expression-based prognostic signatures in lung cancer: ready for clinical use? J Natl Cancer Inst. 2010;102:464-74.

21. Kratz JR, He J, Van Den Eeden SK, Zhu Z-H, Gao W, Pham PT, et al. A practical molecular assay to predict survival in resected non-squamous, non-small-cell lung cancer: development and international validation studies. Lancet. 2012;379:823-32.

22. Gray ES, Rizos H, Reid AL, Boyd SC, Pereira MR, Lo J, et al. Circulating tumor DNA to monitor treatment response and detect acquired resistance in patients with metastatic melanoma. Oncotarget. 2015;6:42008-18.

23. Yang B, Li X, Ren T, Yin Y. Autoantibodies as diagnostic biomarkers for lung cancer: a systematic review. Cell Death Discov. 2019;5:126.

24. Okada M, Nishio W, Sakamoto T, Uchino K, Yuki T, Nakagawa A, et al. Prognostic significance of perioperative serum carcinoembryonic antigen in non-small cell lung cancer: analysis of 1,000 consecutive resections for clinical stage I disease. Ann Thorac Surg. 2004;78:216-21.

25. Karn T, Pusztai L, Ruckhäberle E, Liedtke C, Müller V, Schmidt M, et al. Melanoma antigen family $A$ identified by the bimodality index defines a subset of triple negative breast cancers as candidates for immune response augmentation. Eur J Cancer. 2012;48:12-23.

26. Rousseaux S, Debernardi A, Jacquiau B, Vitte A-L, Vesin A, Nagy-Mignotte $H$, et al. Ectopic activation of germline and placental genes identifies aggressive metastasis-prone lung cancers. Sci Transl Med. 2013;5:186ra66. 
27. Li X-F, Ren P, Shen W-Z, Jin X, Zhang J. The expression, modulation and use of cancer-testis antigens as potential biomarkers for cancer immunotherapy. Am J Transl Res. 2020;12:7002-19.

28. Wang Z, Li Z, Ji H. Direct targeting of $\beta$-catenin in the Wnt signaling pathway: current progress and perspectives. Med Res Rev. 2021. https://doi.org/10.1002/ med.21787.

29. Kim J-H, Kwon J, Lee HW, Kang MC, Yoon H-J, Lee S-T, et al. Protein tyrosine kinase 7 plays a tumor suppressor role by inhibiting ERK and AKT phosphorylation in lung cancer. Oncol Rep. 2014;31:2708-12.

30. Nusse R, Clevers $H$. Wnt/ $\beta$-catenin signaling, disease, and emerging therapeutic modalities. Cell. 2017;169:985-99.

31. Simpson AJG, Caballero OL, Jungbluth A, Chen Y-T, Old LJ. Cancer/testis antigens, gametogenesis and cancer. Nat Rev Cancer. 2005;5:615-25.

32. Sahin U, Oehm P, Derhovanessian E, Jabulowsky RA, Vormehr M, Gold M, et al. An RNA vaccine drives immunity in checkpoint-inhibitor-treated melanoma. Nature. 2020;585:107-12.

33. Semënov MV, Tamai K, Brott BK, Kühl M, Sokol S, He X. Head inducer Dickkopf-1 is a ligand for Wnt coreceptor LRP6. Curr Biol. 2001;11:951-61.

34. Zhang X, Ning Y, Xiao Y, Duan H, Qu G, Liu X, et al. MAEL contributes to gastric cancer progression by promoting ILKAP degradation. Oncotarget. 2017;8:113331-44.

35. Morita K, He S, Nowak RP, Wang J, Zimmerman MW, Fu C, et al. Allosteric activators of protein phosphatase $2 \mathrm{~A}$ display broad antitumor activity mediated by dephosphorylation of MYBL2. Cell. 2020;181:702-15. e20.

36. Peng A, Maller JL. Serine/threonine phosphatases in the DNA damage response and cancer. Oncogene. 2010;29:5977-88.

37. Damelin M, Bankovich A, Bernstein J, Lucas J, Chen L, Williams S, et al. A PTK7targeted antibody-drug conjugate reduces tumor-initiating cells and induces sustained tumor regressions. Sci Transl Med. 2017;9:eaag2611.

38. Gurney A, Axelrod F, Bond CJ, Cain J, Chartier C, Donigan L, et al. Wht pathway inhibition via the targeting of Frizzled receptors results in decreased growth and tumorigenicity of human tumors. Proc Natl Acad Sci USA. 2012;109:11717-22.

39. Kauko O, O'Connor CM, Kulesskiy E, Sangodkar J, Aakula A, Izadmehr S, et al. PP2A inhibition is a druggable MEK inhibitor resistance mechanism in KRASmutant lung cancer cells. Sci Transl Med. 2018;10:eaaq1093. https://doi.org/ 10.1126/scitranslmed.aaq1093.

40. Jeong AL, Han S, Lee S, Su Park J, Lu Y, Yu S, et al. Patient derived mutation W257G of PPP2R1A enhances cancer cell migration through SRC-JNK-c-Jun pathway. Sci Rep. 2016;6:27391.

41. Da Gama Duarte J, Peyper JM, Blackburn JM. B cells and antibody production in melanoma. Mamm Genome J Int Mamm Genome Soc. 2018;29:790-805.

42. Zaenker P, Lo J, Pearce R, Cantwell $P$, Cowell $L$, Lee $M$, et al. A diagnostic autoantibody signature for primary cutaneous melanoma. Oncotarget. 2018;9:30539-51.

43. Zaenker P, Gray ES, Ziman MR. Autoantibody production in cancer-the humoral immune response toward autologous antigens in cancer patients. Autoimmun Rev. 2016;15:477-83.

44. Garcia J, Faca V, Jarzembowski J, Zhang Q, Park J, Hanash S. Comprehensive profiling of the cell surface proteome of Sy5Y neuroblastoma cells yields a subset of proteins associated with tumor differentiation. J Proteome Res. 2009;8:3791-6.

\section{ACKNOWLEDGEMENTS}

We would like to acknowledge the assistance of Hollie Bancroft in locating and procuring samples and metadata. JMB thanks the National Research Foundation (South Africa) for a SARChl grant. This article has been written in accordance with the REMARK guidelines as outlined on the EQUATOR network (see attached REMARK checklist).

\section{AUTHOR CONTRIBUTIONS}

AJP and GWM designed the experimental plan. AJP carried out sample procurement, processing and data analysis. JMB designed the Immunome protein array platform and the data pre-processing pipeline. TM and JMB contributed to the data analysis. AJP and GWM interpreted the results and constructed and designed the manuscript. $A G R, B N, T M$ and JMB provided constructive feedback on the design and layout of the manuscript.

\section{FUNDING}

Funding for this work was provided through an Immunology Grant from the University of Birmingham Development and Alumni Relations Office (DARO) Charitable Fund. AJP was funded through a Cancer Research UK-TRACERx fellowship that helped facilitate the execution of this work.

\section{ETHICS APPROVAL AND CONSENT TO PARTICIPATE}

All participants provided informed consent to participate in future translational studies when they were initially recruited, previously approved by the West Midlands -Solihull Research Ethics Committee (Cancer of the Lung Biomarkers (CLUB): REC reference: 04/Q2704/34). The study had National Cancer Research Network (NCRN) approval and was an NCRN portfolio study. The study was performed in full accordance with the Declaration of Helsinki.

\section{CONSENT TO PUBLISH}

No individual patient-identifiable data was used, all were pseudonymised and full consent was gained as part of the recruitment process.

\section{COMPETING INTERESTS}

JMB is a Director of Sengenics Corporation, who commercialise the Immunome protein array.

\section{ADDITIONAL INFORMATION}

Supplementary information The online version contains supplementary material available at https://doi.org/10.1038/s41416-021-01572-x.

Correspondence and requests for materials should be addressed to Gary W. Middleton.

Reprints and permission information is available at http://www.nature.com/ reprints

Publisher's note Springer Nature remains neutral with regard to jurisdictional claims in published maps and institutional affiliations.

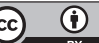

Open Access This article is licensed under a Creative Commons Attribution 4.0 International License, which permits use, sharing, adaptation, distribution and reproduction in any medium or format, as long as you give appropriate credit to the original author(s) and the source, provide a link to the Creative Commons license, and indicate if changes were made. The images or other third party material in this article are included in the article's Creative Commons license, unless indicated otherwise in a credit line to the material. If material is not included in the article's Creative Commons license and your intended use is not permitted by statutory regulation or exceeds the permitted use, you will need to obtain permission directly from the copyright holder. To view a copy of this license, visit http://creativecommons. org/licenses/by/4.0/.

(c) The Author(s) 2021 